\title{
Modelling of a marine glacier and ice-sheet-ice-shelf transition zone based on asymptotic analysis
}

\author{
Vladimir A. Chugunov and Alexander V. Wilchinsky \\ Department of Applied Mathematics, Kazan State University, Kazan 420008, Tatarstan, Russia
}

\begin{abstract}
All parts of a two-dimensional, isothermal, stationary marine glacier (grounded ice sheet, ice shelf and transition zone) with constant viscosity are analysed by perturbation methods. In so doing, all zones of different flow patterns can be considered separately. Correlations between spatial scales for all parts can be expressed in terms of the typical ice-surface slope distant from the ocean, which reflects exterior conditions of the glacier's existence. In considering the ice-sheet-ice-shelf transition zone, a small parameter characterizing the difference between ice and water densities is used. Such an analysis allows us to find boundary conditions at the grounding line for the grounded ice mass. Glacier-surface profiles are determined by numerical methods. The grounding-line position found by using the boundary conditions derived in this paper differs from that obtained by using Thomas and Bentley's (1978) boundary conditions by about $10 \%$ of the grounded ice-stream length.
\end{abstract}

\section{INTRODUCTION}

Modelling the dynamics of the grounded part of a marine ice sheet requires the imposition of boundary conditions at its terminus (grounding line). At the same time, the position of the terminus is sensitive to changes of such conditions (Lingle, 1984). Usually, it is assumed that the ice has flotation thickness at the grounding line and the terminus position is determined by using the massbalance equation reduced by the shallow-ice approximation (Thomas and Bentley, 1978). Salamatin (1984) modified this relation by using an unknown parameter, which characterizes the deviation of the reduced normal deviatoric stress from its exact value. However, the mathematical algorithm used to find this parameter is obscure. On the other hand, because the styles of flow in the grounded part of the glacier and in the ice shelf are essentially different (a shearing flow and a plug flow, respectively), it follows that in the ice-sheet-ice-shelf transition zone neither shear stress nor normal stress can be neglected. Hence, the problem of finding the stress fields in the transition zone arises and hence determining strict mathematically derived effective boundary conditions for the ice sheet at the grounding line.

Determination of the ice upper-surface profile in the vicinity of the grounding line should lead to an algorithm for detecting the line position by examination of the upper-surface characteristics. This problem was also considered in the paper of Barcilon and MacAyeal (1993), in which the problem of fluid flow down an inclined plane with an abrupt change of boundary conditions on it (no-slip-free slip) is considered. Although the problem they considered was somewhat different than that considered here, the existence of a local minimum of the upper-surface elevation is confirmed by the results of the present paper.

In this work, we study an idealized model of a twodimensional, stationary, isothermal glacier in all parts of its ice flow: grounded ice sheet, ice shelf and transition zone. Here, ice is considered as a Newtonian fluid. Although the ice flow in the grounded glacier and the ice shelf has been closely examined by many researchers (e.g. Sanderson, 1979; Hutter, 1983), the dynamics of the icesheet-ice-shelf transition zone is poorly understood and the main approach to the problem has been numerical (Lestringant, 1994).

\section{EQUATIONS}

\subsection{Notation}

The following notation will be used: $(x, z)$ are the horizontal and vertical spatial coordinates; $\pi$ is the excess pressure, associated with the pressure $p$ by the relation $\pi=p-\rho_{\mathrm{i}} g(\ell-z) ; \ell(x)$ is the upper-surface elevation; $\ell^{\prime}=\mathrm{d} \ell / \mathrm{d} x, \quad z_{0}(x)$ is the lower-surface profile, which 


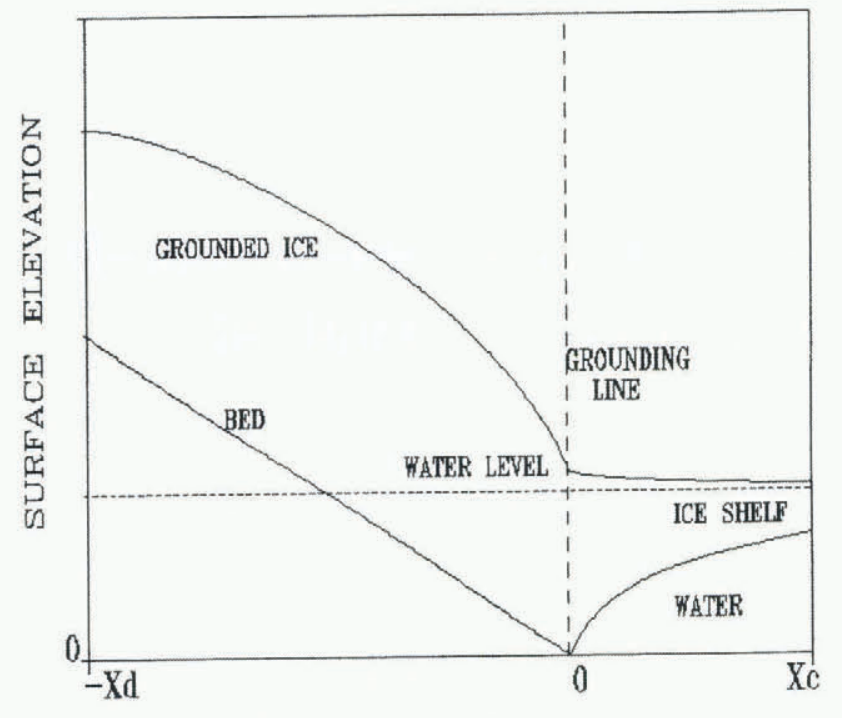

HORIZONTAL COORDINATE X

Fig. 1. Schematic representation of a marine glacier consisting of different parts (grounded ice sheet, ice shelf, ice-sheet ice-shelf transition zone) which are characterized by specific flow patterns (shearing, plug and transition). The origin of the reclangular lefthand coordinate system is set at the bed at the grounding line.

coincides with the bed elevation when $x<0 ; \tau_{x x}, \tau_{z z}, \tau_{x z}$ are the components of the stress deviator; $(u, w)$ is the velocity vector; $\eta$ is the viscosity; $f^{+},\left(-f^{-}\right)$are the accumulation rates on the upper and the lower surfaces, respectively $\left(f^{-}=0, x<0\right) ; g$ is the acceleration due to gravity; $\rho_{\mathrm{i}}, \rho_{\mathrm{w}}$ are the ice and water densities; $k_{\rho}=\rho_{\mathrm{i}} / \rho_{\mathrm{w}}$; $\delta=1-\rho_{\mathrm{i}} / \rho_{\mathrm{w}} ; z^{*}$ is the water level; $[v]$ is the scale magnitude of $v$ (for any variable $v$ ) in the grounded part of the glacier; $\left(-x_{\mathrm{d}}\right)$ is the position of the ice divide; $x_{\mathrm{c}}$ is the position of the ice front; $\sigma$ and $\tau$ are the resultant normal and shear forces.

\subsection{Setting the problem}

Considering a two-dimensional glacier merging into the ocean (Fig. 1), let us place the origin of a rectangular righthand coordinate system ( $z$ axis is directed vertically upwards) at the bed at the grounding line. Then, the regions $\left(-x_{\mathrm{d}}\right)<x<0$ and $0<x<x_{\mathrm{c}}$, where $\left(-x_{\mathrm{d}}\right), x_{\mathrm{c}}$ are the positions of the ice divide (dome) and the ice front, define the grounded and floating parts of the glacier, respectively.

Stationary ice flow, assuming incompressibility and constant viscosity of the ice, can be described by the following Stokes' equations:

$$
\begin{aligned}
-\frac{\partial \pi}{\partial x}+\frac{\partial \tau_{x x}}{\partial x}+\frac{\partial \tau_{x z}}{\partial z} & =\rho_{\mathrm{i}} g \ell^{\prime} ; \\
-\frac{\partial \pi}{\partial z}+\frac{\partial \tau_{z z}}{\partial z}+\frac{\partial \tau_{x z}}{\partial x} & =0 ; \\
\tau_{x x}+\tau_{z z} & =0 ; \\
\tau_{x x}=2 \eta \frac{\partial u}{\partial x} ; \tau_{z z}=2 \eta \frac{\partial w}{\partial z} ; \tau_{x z} & =\eta\left(\frac{\partial u}{\partial z}+\frac{\partial w}{\partial x}\right) ; \\
-x_{\mathrm{d}}<x<x_{c}, z_{0}<z & <\ell .
\end{aligned}
$$

The above set of equations must be completed by the following boundary conditions:

The glacier upper surface is stress-free and the kinematic boundary condition is used to determine its profile:

$$
z=\ell(x):\left\{\begin{array}{l}
\tau_{x z}=-\pi \frac{2 \ell^{\prime}}{1+\ell^{\prime 2}} ; \quad \tau_{x x}=-\pi \frac{1-\ell^{\prime 2}}{1+\ell^{\prime 2}} ; \\
-u \ell^{\prime}+w+f^{+}=0 .
\end{array}\right.
$$

The no-slip condition at the bed may be assumed for the cold Antarctic ice sheet, where sliding is negligible (Fowler, 1981):

$$
z=z_{0}(x), x<0: u=0, w=0 .
$$

At the submerged lower surface of the ice shelf, the shear stress is zero, the normal stress is equal to the water pressure and a kinematic boundary condition is applied:

$$
\begin{gathered}
z=z_{0}(x), x>0: \\
\left\{\begin{array}{l}
\tau_{x z}=-\left[\rho_{\mathrm{i}} g\left(\ell-z_{0}\right)-\rho_{\mathrm{w}} g\left(z^{*}-z_{0}\right)+\pi\right] \frac{2 z_{0}^{\prime}}{1+z_{0}^{\prime 2}} \\
\tau_{x x}=-\left[\rho_{\mathrm{i}} g\left(\ell-z_{0}\right)-\rho_{\mathrm{w}} g\left(z^{*}-z_{0}\right)+\pi\right] \frac{1-z_{0}^{\prime 2}}{1+z_{0}^{\prime 2}} \\
-u z_{0}^{\prime}+w+f^{-}=0
\end{array}\right.
\end{gathered}
$$

At the ice divide, the mass flux is zero and the fluid flow is symmetric:

$$
x=-x_{\mathrm{d}}: u=0, \frac{\partial w}{\partial x}=0 .
$$

At the ice front, where calving takes place, it is sufficient to impose the value of the resultant normal stress in the ice, whose magnitude in water is known (Weertman, 1957):

$$
\int_{z_{0}}^{\ell}\left(-p+\tau_{x x}\right) \mathrm{d} z=-\frac{\rho_{\mathrm{w}} g}{2}\left(z^{*}-z_{0}\right)^{2} .
$$

As we will see later, the solution of the problem does not depend on the ice-front position, whose existence is necessary only to derive the relation (6). Thus, let us assume that there is no calving and that $x_{c}$ is the point of zero mass flux: $q\left(x_{\mathrm{c}}\right)=\int_{-x_{\mathrm{d}}}^{x_{\mathrm{c}}}\left(f^{+}-f^{-}\right) \mathrm{d} x=0$, or $x_{\mathrm{c}}=+\infty$ when the mass flux is positive everywhere: $q(x)>0, \forall x>0$.

We introduce the resultant forces at an arbitrary crosssection :

$$
\sigma=\int_{z_{0}}^{\ell}\left(-p+\tau_{x x}\right) \mathrm{d} z, \quad \tau=\int_{z_{0}}^{\ell} \tau_{x z} \mathrm{~d} z .
$$

Integrating Equation (1) with respect to $z$ from $z_{0}$ to $\ell$ and taking into account the boundary conditions (2), (3) and (4), we obtain the following equations:

$$
\begin{array}{ll}
\frac{\mathrm{d} \sigma}{\mathrm{d} x}=p\left(x, z_{0}\right) z_{0}^{\prime}+\left(1+z_{0}^{\prime 2}\right) \eta \frac{\partial u}{\partial z}\left(x, z_{0}\right), & x<0 ; \\
\frac{\mathrm{d} \sigma}{\mathrm{d} x}=\rho_{\mathrm{w}} g\left(z^{*}-z_{0}\right) z_{0}^{\prime}, & x>0 ; \\
\frac{\mathrm{d} \tau}{\mathrm{d} x}=\rho_{\mathrm{i}} g\left(\ell-z_{0}\right)-\rho_{\mathrm{w}} g\left(z^{*}-z_{0}\right), & x>0 .
\end{array}
$$


Integrating Equation (8) with respect to $x$ and using Equation (6), we have for $x>0$ :

$$
\sigma=-\frac{\rho_{\mathrm{w}} g}{2}\left(z^{*}-z_{0}\right)^{2} .
$$

\subsection{Non-dimensionalization}

Far from the ocean, the ice flow is described by the shallow-ice approximation (Hutter, 1983), where the shear stress is much larger than the normal stress. Nondimensionalizing the governing equations, with the following scales found by Salamatin and Mazo (1984) on the basis of similarity theory:

$$
\begin{aligned}
{[w]=\left[f^{ \pm}\right] } & =\frac{1}{x_{\mathrm{d}}} \int_{-x_{\mathrm{d}}}^{0} f^{+} \mathrm{d} x ; \quad[u]=\frac{[x][w]}{[z]} ; \quad\left[\tau_{x, x}\right]=\frac{\eta[w]}{[z]} ; \\
{\left[\tau_{x z}\right] } & =\frac{\eta[u]}{[z]} ; \quad[\ell]=\left[z_{0}\right]=\left[z^{*}\right]=[z] ; \quad[x]=x_{\mathrm{d}} ; \\
{[\pi] } & =\rho_{\mathrm{i}} g[z] ; \quad[\tau]=[z]\left[\tau_{x z}\right] ; \quad[z]=\left(\frac{[w] \eta[x]^{2}}{\rho_{\mathrm{i}} g}\right)^{\frac{1}{+}} ; \\
\epsilon & =\frac{[z]}{[x]} ; \quad k_{\rho}=\frac{\rho_{\mathrm{i}}}{\rho_{\mathrm{w}}} ; \quad[\sigma]=\rho_{\mathrm{i}} g[z]^{2},
\end{aligned}
$$

we obtain the following equations (capital letters denote the dimensionless variables $\left.T_{x x}=\tau_{x x} /\left[\tau_{x x}\right]\right)$ :

$$
\begin{gathered}
-\frac{\partial \Pi}{\partial X}+\epsilon^{2} \frac{\partial T_{x x}}{\partial X}+\frac{\partial T_{x z}}{\partial Z}=L^{\prime} ; \\
-\frac{\partial \Pi}{\partial Z}+\epsilon^{2}\left(\frac{\partial T_{z z}}{\partial Z}+\frac{\partial T_{x z}}{\partial X}\right)=0 ; \\
T_{x x}+T_{z z}=0 ;
\end{gathered}
$$

$$
T_{x x}=2 \frac{\partial U}{\partial X} ; T_{z z}=2 \frac{\partial W}{\partial Z} ; T_{x z}=\frac{\partial U}{\partial Z}+\epsilon^{2} \frac{\partial W}{\partial X} ;
$$$$
-1<X<X_{\mathrm{c}}, \quad Z_{0}<Z<L ;
$$

$Z=L(X):$

$$
\left\{\begin{array}{l}
T_{x z}=-\Pi \frac{2 L^{\prime}}{1+\epsilon^{2} L^{\prime 2}} ; \epsilon^{2} T_{x x}=-\Pi \frac{1-\epsilon^{2} L^{\prime 2}}{1+\epsilon^{2} L^{\prime 2}} ; \\
-U L^{\prime}+W+F^{+}=0
\end{array}\right.
$$

$Z=Z_{0}(X), X<0: U=0, W=0$;

$Z=Z_{0}(X), X>0$ :

$$
\left\{\begin{array}{l}
T_{x z}=-\left[L-Z_{0}-\frac{1}{k_{p}}\left(Z^{*}-Z_{0}\right)+\Pi\right] \frac{2 Z_{0}^{\prime}}{1+\epsilon^{2} Z_{0}^{\prime 2}} \\
\epsilon^{2} T_{x x}=-\left[L-Z_{0}-\frac{1}{k_{p}}\left(Z^{*}-Z_{0}\right)+\Pi\right] \frac{1-\epsilon^{2} Z_{0}^{\prime 2}}{1+\epsilon^{2} Z_{0}^{\prime 2}} \\
-U Z_{0}^{\prime}+W+F^{-}=0
\end{array}\right.
$$

$X=-1: U=0, \frac{\partial W}{\partial X}=0$;

$k_{\rho}=\frac{\rho_{\mathrm{i}}}{\rho_{\mathrm{w}}}$.

Most of the cold marine glaciers are characterized by a value of the parameter $\epsilon \ll 1$. This parameter is the typical ice-surface slope and reflects exterior conditions of the glacier's existence, such as the scales of accumulation rate, its gradient, bed slope and water level.

In order to analyse the problem in Equations (12)
(16), we introduce the stream function $\psi$ :

$$
U=\frac{\partial \psi}{\partial Z} ; \quad W=-\frac{\partial \psi}{\partial X} .
$$

From the Equations (12) and boundary conditions (13)(16), we obtain the following set of equations to determine $\psi$ when $L$ and $Z_{0}$ are given:

$$
\begin{gathered}
\epsilon^{4} \frac{\partial^{4} \psi}{\partial X^{4}}+2 \epsilon^{2} \frac{\partial^{4} \psi}{\partial X^{2} \partial Z^{2}}+\frac{\partial^{4} \psi}{\partial Z^{4}}=0 ; \\
-1<X<X_{\mathrm{c}}, \quad Z_{0}<Z<L ;
\end{gathered}
$$

$Z=L(X):$

$\left\{\begin{array}{l}{\left[\frac{\partial^{2} \psi}{\partial Z^{2}}-\epsilon^{2} \frac{\partial^{2} \psi}{\partial X^{2}}\right]\left(1-\epsilon^{2} L^{\prime 2}\right)=4 \epsilon^{2} L^{\prime} \frac{\partial^{2} \psi}{\partial X \partial Z}} \\ \psi=\int_{-1}^{X} F^{+} \mathrm{d} X ;\end{array}\right.$

$Z=Z_{0}(X), X<0: \psi=0, \frac{\partial \psi}{\partial Z}=0 ;$

$Z=Z_{0}(X), X>0$ :

$\left\{\begin{array}{l}{\left[\frac{\partial^{2} \psi}{\partial Z^{2}}-\epsilon^{2} \frac{\partial^{2} \psi}{\partial X^{2}}\right]\left(1-\epsilon^{2} Z_{0}^{\prime 2}\right)=4 \epsilon^{2} Z_{0}^{\prime} \frac{\partial^{2} \psi}{\partial X \partial Z}} \\ \psi=\int_{0}^{X} F^{-} \mathrm{d} X\end{array}\right.$

$X=-1: \frac{\partial \psi}{\partial Z}=0, \frac{\partial^{2} \psi}{\partial X^{2}}=0$.

Having determined $\psi$, we can find all the unknown fields except the excess pressure $\Pi$ as well as $L$ and $Z_{0}(X>0)$. In order to find $\Pi$, we use the first equation of $(12)$ integrated from $Z$ to $L$ and the boundary conditions (13) to obtain, in terms of $\psi$,

$$
\begin{aligned}
& \Pi(X, Z)= \\
& -\epsilon^{2}\left[2 \frac{\partial^{2} \psi}{\partial X \partial Z}+\frac{\partial}{\partial X} \int_{Z}^{L}\left(\frac{\partial^{2} \psi}{\partial Z^{2}}-\epsilon^{2} \frac{\partial^{2} \psi}{\partial X^{2}}\right) \mathrm{d} Z\right], \forall X .
\end{aligned}
$$

Let us define

then

$$
\tilde{T}(X, Z)=\int_{Z}^{L}\left(\frac{\partial^{2} \psi}{\partial Z^{2}}-\epsilon^{2} \frac{\partial^{2} \psi}{\partial X^{2}}\right) \mathrm{d} Z ;
$$

$$
T(X)=\frac{\tau}{[\tau]}=\widetilde{T}\left(X, Z_{0}\right) .
$$

Because the relations ( 8 and (9) are derived by integration of Equations (1) with respect to $z$ using the boundary conditions on the upper and lower surfaces, some of which were not used in the system of Equations (17)-(21), these relations can be considered as the equations to determine $L$ and $Z_{0}$, when $X>0$. Converting Equations (8) and (9) into dimensionless form and substituting for $\Pi$ via Equation (22), we obtain the following equations for $L$ and $Z_{0}$, when $X>0$ :

$$
\begin{gathered}
\frac{\left(L-Z_{0}\right)^{2}}{2}-\frac{\left(Z^{*}-Z_{0}\right)^{2}}{2 k_{\rho}}=\epsilon^{2} \int_{Z_{01}}^{L}\left[4 \frac{\partial^{2} \psi}{\partial X \partial Z}+\frac{\partial \tilde{T}}{\partial X}\right] \mathrm{d} Z, \\
X>0 ; \\
Z_{0}-Z^{*}+k_{\rho}\left(L-Z_{0}\right)=k_{\rho} \epsilon^{2} \frac{\mathrm{d} T}{\mathrm{~d} X}, \quad X>0 \\
X=0: Z_{0}=0 .
\end{gathered}
$$


In order to determine $L$ in the region $X<0$, we integrate Equation (7) with respect to $X$ from $X$ to 0 . Since $\Sigma(0)=\sigma(0) /[\sigma]=-Z^{* 2} / 2 k_{\rho}$, this yields in dimensionless form:

$$
\begin{aligned}
& \frac{\left(L-Z_{0}\right)^{2}}{2}-\frac{Z^{* 2}}{2 k_{\rho}}= \\
& \epsilon^{2} \int_{Z_{0}}^{L}\left(4 \frac{\partial^{2} \psi}{\partial X \partial Z}+\frac{\partial \tilde{T}}{\partial X}\right) \mathrm{d} Z+\int_{X}^{0}\left(L-Z_{0}\right) Z_{0}^{\prime} \mathrm{d} X \\
& +\int_{X}^{0}\left[\Pi\left(X, Z_{0}\right) Z_{0}^{\prime}+\left(1+\epsilon^{2} Z_{0}^{\prime 2}\right) \frac{\partial^{2} \psi}{\partial Z^{2}}\left(X, Z_{0}\right)\right] \mathrm{d} X, \\
& X<0 .
\end{aligned}
$$

\section{FAR-FIELD SOLUTIONS}

\subsection{Far-field solution for the grounded ice mass}

We seek an expansion for $\psi^{(g)}, L^{(g)}$ in the far field for the region $X<0$ as an asymptotic series in $\epsilon$ :

$$
\begin{aligned}
& \psi^{(g)}=\psi_{0}^{(g)}+O\left(\epsilon^{2}\right) ; \\
& L^{(g)}=L_{0}^{(g)}+O\left(\epsilon^{2}\right) .
\end{aligned}
$$

Then, from Equations (17)-(21) we derive

$$
\psi_{0}^{(g)}=\frac{Q\left(Z-Z_{0}\right)^{2}\left(3 L_{0}^{(g)}-2 Z_{0}-Z\right)}{2\left(L_{0}^{(g)}-Z_{0}\right)^{3}}
$$

where $Q=\int_{-1}^{X} F^{+} \mathrm{d} X$. This solution describes Poiseuille flow.

From Equation (25), we have for $L_{0}^{(g)}$ that

$$
\begin{aligned}
\frac{\left(L_{0}^{(g)}-Z_{0}\right)^{2}}{2}-\frac{Z^{* 2}}{2 k_{\rho}}= & \int_{X}^{0}\left(L_{0}^{(g)}-Z_{0}\right) Z_{0}^{\prime} \mathrm{d} X \\
& +\int_{X}^{0} \frac{\partial^{2} \psi}{\partial Z^{2}}\left(X, Z_{0}\right) \mathrm{d} X .
\end{aligned}
$$

Differentiating this equation with respect to $X$, we find

$$
\frac{\mathrm{d} L_{0}^{(g)}}{\mathrm{d} X}=-\frac{3 Q}{\left(L_{0}^{(g)}-Z_{0}\right)^{3}} .
$$

It should be noted that $\psi_{0}^{(g)}$ only satisfies boundary conditions at the bed and the upper surface of the glacier, since all the $X$ derivatives in the problem for $\psi_{0}^{(g)}$ were omitted. These boundary conditions imply that mass flux at the ice divide is zero. Equation (26) allows us to determine the upper-surface profile of the glacier far from the grounding line. However, in order to derive a unique solution to the far-field problem, it is necessary to determine the far-field surface elevation at the grounding line $L_{0}^{(g)}(0)$, as well as the magnitude of $x_{\mathrm{d}}$, which is also unknown. These parameters are to be determined from the matching procedure.

In general, we cannot expect the stream function we have found to satisfy all the conditions at the ice divide, since the shear stress there is zero, although we have assumed that shear stresses dominate normal stresses in the shallow-ice approximation. A local expansion near the dome may be necessary but it can be shown, at least for constant viscosity, that the far-field solution is in fact valid up to the divide. Details of this derivation will be given elsewhere.

\subsection{Far-field solution for the ice shelf}

If $X>0$, and we search for the far-field solution $\psi^{(s)}, L^{(s)}, Z_{0}^{(s)}$ to the set of Equations (17)-(21), (23) and (24) in the form of an asymptotic series in $\epsilon$, the leading-order term of the ice thickness proves to be zero. Hence, for the ice shelf, it is necessary to introduce its own far-field variables. In this theoretical study, where no calving is assumed and the ice shelf can be of infinite length, we suppose that far from the grounding line the scale of the longitudinal coordinate is the same as that for the grounded ice sheet. Hence, only the scale of the vertical coordinate must change. Only one transformation gives us non-zero leading-order ice thickness in the ice shelf, n a m e ly $\widetilde{Z}=Z / \epsilon, \widetilde{L}=L / \epsilon, \widetilde{Z_{0}}=Z_{0} / \epsilon, \widetilde{Z^{*}}=Z^{*} / \epsilon$. Therefore, the coordinate $\widetilde{Z}$ must be taken as the farfield vertical coordinate for the ice shelf. Notice that, in terms of the grounded ice-sheet scale, the ice-shelf thickness is of order $\epsilon$, which dimensionally corresponds to a scale of only a metre or so. It is to be emphasized that this scale is only appropriate thousands of kilometres from the grounding line and, in reality, calving of icebergs causes ice shelves to be much shorter (and hence thicker). Expanding the unknown functions as power series in $\epsilon$ :

$$
\begin{aligned}
& {\psi^{(s)}}^{(s)}\left(\psi_{0}^{(s)}+O(\epsilon) ;\right. \\
& \widetilde{L^{(s)}}=\widetilde{L_{0}^{(s)}}+O(\epsilon) ; \\
& \widetilde{Z_{0}^{(s)}}=\widetilde{Z_{00}^{(s)}}+O(\epsilon),
\end{aligned}
$$

we can find a far-field solution in the following form (for convenience, all the formulae are written in the far-field variables of the grounded part):

$$
\psi_{0}^{(s)}=\frac{Q}{H}\left(Z-Z^{*}\right)+k_{\rho} Q+\int_{0}^{X} F^{-} \mathrm{d} X,
$$

where

$$
\begin{gathered}
Q=1+\int_{0}^{X} F \mathrm{~d} X, F=F^{+}-F^{-}, \quad H=L_{0}^{(s)}-Z_{00}^{(s)}, \\
H=\epsilon Q\left(A+\frac{\delta}{4} \int_{0}^{X} Q \mathrm{~d} X\right)^{-\frac{1}{2}}, \quad L_{0}^{(s)}=Z^{*}+\delta H, \\
Z_{00}^{(s)}=Z^{*}-k_{\rho} H, \delta=1-\frac{\rho_{\mathrm{i}}}{\rho_{\mathrm{w}}},
\end{gathered}
$$

and $A$ is constant.

The far-field solution we have found coincides with that derived by Van der Veen (1983). The constant $A$ is to be determined from the matching procedure.

As mentioned above, we note that for $X=O(1)$, that is, at a distance from the grounding line comparable with the grounded ice-sheet length, $H=O(\epsilon)$. However, the value of the constant $A$ found by the matching procedure turns out to be such that $H$ is $O\left((\epsilon / \delta)^{\frac{1}{3}}\right)$ in the vicinity of the grounding line. Numerically, this is around 0.2 , corresponding to a thickness in the vicinity of $500 \mathrm{~m}$, as is observed.

If we compare the far-field solutions for the stream function in the different regions, it is clear that these are essentially different and cannot be matched. Therefore, a 
near-field solution in the vicinity of the grounding line is necessary to match the solution for the grounded part to that for the ice shelf. This near-field solution defines the ice-sheet-ice-shelf transition zone.

\section{NEAR-FIELD SOLUTION FOR THE TRANSITION ZONE}

\subsection{Equations}

In the original coordinate system, the gradients of the fields are proportional to $\epsilon$, whose value is imposed by the external conditions, such as the scales of accumulation rate, its gradient and the bed slope. But, in the ice-sheetice-shelf transition zone, the velocity gradient is imposed mainly by the transition from no-slip to free-floating. This allows us to make the assumption that the longitudinal scale of the transition zone is the same as the vertical one; the ratio between these does not depend on $\epsilon$.

Denoting the ice thickness at the grounding line in the scales of the grounded part by $L_{0}$, let us introduce the near-field coordinates and variables:

$$
\begin{gathered}
\xi=X / \epsilon L_{0}, \quad y=Z / L_{0}, \quad \chi=L / L_{0}, \quad \vartheta=Z_{0} / L_{0}, \\
\vartheta^{*}=Z^{*} / L_{0}, \quad h=\chi-\vartheta .
\end{gathered}
$$

Rewriting Equations (17)-(21) in the new variables and neglecting bed roughness, we derive

$$
\frac{\partial^{4} \psi}{\partial \xi^{4}}+2 \frac{\partial^{4} \psi}{\partial \xi^{2} \partial y^{2}}+\frac{\partial^{4} \psi}{\partial y^{4}}=0,-\infty<\xi<+\infty, \vartheta<y<\chi
$$

$y=\chi(\xi):$

$$
\left\{\begin{array}{l}
{\left[\frac{\partial^{2} \psi}{\partial y^{2}}-\frac{\partial^{2} \psi}{\partial \xi^{2}}\right]\left(1-\chi^{\prime 2}\right)=4 \chi^{\prime} \frac{\partial^{2} \psi}{\partial \xi \partial y}} \\
\psi=1+\epsilon L_{0} \int_{0}^{\xi} F^{+} \mathrm{d} \xi
\end{array}\right.
$$

$y=\vartheta(\xi)=Z_{0}^{\prime}(0) \epsilon \xi+O\left(\epsilon^{2}\right), \xi<0: \psi=0, \frac{\partial \psi}{\partial y}=0 ;$

$y=\vartheta(\xi), \xi>0$ :

$$
\begin{aligned}
& \left\{\begin{array}{l}
{\left[\frac{\partial^{2} \psi}{\partial y^{2}}-\frac{\partial^{2} \psi}{\partial \xi^{2}}\right]\left(1-\vartheta^{\prime 2}\right)=4 \vartheta^{\prime} \frac{\partial^{2} \psi}{\partial \xi \partial y}} \\
\psi=\epsilon L_{0} \int_{0}^{\xi} F^{-} \mathrm{d} \xi
\end{array}\right. \\
& \xi \rightarrow \pm \infty: \psi<\infty \text {. }
\end{aligned}
$$

The conditions (32) are sufficient for matching the near-field stream function with those of the far fields. Equations (28)-(32) define $\psi$. For the functions $\chi$ and $h$, it follows from Equations (23)-(25) that we have the following equations:

$$
\begin{aligned}
& \left\{\begin{array}{lr}
\frac{\chi^{2}}{2}-\chi \vartheta-\frac{\vartheta^{* 2}}{2 k_{\rho}}=\delta \beta^{-1} \int_{\vartheta}^{\chi} B \mathrm{~d} y+\delta \beta^{-1} \int_{\xi}^{0} \frac{\partial^{2} \psi}{\partial y^{2}}(\xi, \vartheta) \mathrm{d} \xi+O(\epsilon) ; \\
\vartheta(\xi)=Z_{0}^{\prime}(0) \epsilon \xi+O\left(\epsilon^{2}\right), & \xi<0 ; \\
\xi=0: \chi=1 ;
\end{array}\right. \\
& \begin{cases}\frac{h^{2}}{2}+k_{\rho} h \beta^{-1} \frac{\mathrm{d} T}{\mathrm{~d} \xi}-\frac{k_{\rho}}{2} \delta \beta^{-2}\left(\frac{\mathrm{d} T}{\mathrm{~d} \xi}\right)^{2}=\beta^{-1} \int_{\vartheta}^{\chi} B \mathrm{~d} y, & \xi>0 ; \\
\xi=0: h=1 ;\end{cases}
\end{aligned}
$$

$$
\begin{array}{cc}
B(\psi, \chi, \xi, y)=4 \frac{\partial^{2} \psi}{\partial \xi \partial y}+\frac{\partial}{\partial \xi} \int_{y}^{\chi}\left(\frac{\partial^{2} \psi}{\partial y^{2}}-\frac{\partial^{2} \psi}{\partial \xi^{2}}\right) \mathrm{d} y, & \forall \xi ; \\
T(\psi, \chi, \vartheta, \xi)=\int_{\vartheta}^{\chi}\left(\frac{\partial^{2} \psi}{\partial y^{2}}-\frac{\partial^{2} \psi}{\partial \xi^{2}}\right) \mathrm{d} y, & \forall \xi ; \\
\beta=\frac{\delta L_{0}^{3}}{\epsilon} . &
\end{array}
$$

From Equations (23) and (24), $\chi, \vartheta$ and $\vartheta^{*}$ can be expressed via $h$ and $T$ :

$$
\begin{aligned}
& \vartheta=\vartheta^{*}-k_{\rho} h+\delta \beta^{-1} k_{\rho} \frac{\mathrm{d} T}{\mathrm{~d} \xi}, \quad \xi>0 ; \\
& \chi=\vartheta^{*}+\delta h+\delta \beta^{-1} k_{\rho} \frac{\mathrm{d} T}{\mathrm{~d} \xi}, \quad \xi>0 ; \\
& \vartheta^{*}=k_{\rho}-\delta \beta^{-1} k_{\rho} \frac{\mathrm{d} T}{\mathrm{~d} \xi}(0) .
\end{aligned}
$$

Asymptotic analysis of Equation (34) shows that

$$
\begin{aligned}
& \text { if } \beta \rightarrow 0 \text { as } \epsilon \rightarrow 0 \text {, then } \frac{\partial T}{\partial \xi} \rightarrow 0 \text { as } \epsilon \rightarrow 0 \text {, } \\
& \text { because } h=O(1) \text {; } \\
& \text { if } \beta \rightarrow \infty \text { as } \epsilon \rightarrow 0 \text {, then } \frac{\partial T}{\partial \xi} \rightarrow \infty \text { as } \epsilon \rightarrow 0 \text {. }
\end{aligned}
$$

These cases contradict the assumption of finite longitudinal scale of the transition zone.

Hence, we have $\beta=O(1)$, as $\epsilon \rightarrow 0$, so that $\beta=$ constant $+O(\epsilon)$. This is a rigorous result of the asymptotic analysis of Equation (34). It is also true for non-isothermal glaciers or ice sheets with non-zero bed roughness, as the former equation does not change, except for the expressions for $T$ and $B$. Then we can find the scale of the ice thickness at the grounding line $\Delta_{0}$, taking $\beta=1$ (for example) in Equation (35):

$$
\frac{\Delta_{0}^{3}}{h_{0}^{3}}=\frac{\epsilon}{\delta} \text {, or } \Delta_{0}=\left(\frac{\eta q_{0}}{\rho_{\mathrm{i}} g \delta}\right)^{\frac{1}{3}}, \quad \text { where } q_{0}=\int_{-x_{\mathrm{d}}}^{0} f^{+} \mathrm{d} x \text {. }
$$

As a result, we can conclude that, assuming the average grounded ice thickness is $O(1)$ (corresponding to $3000 \mathrm{~m}$ ), the typical ice thicknesses for the transition zone and the ice shelf (sufficiently far from the grounding line) will be $O\left((\epsilon / \delta)^{\frac{1}{3}}\right)$ (corresponding to a thickness of $800 \mathrm{~m}$ ) and $O(\epsilon)$ (corresponding to $6 \mathrm{~m}$ ), respectively, when $\epsilon=2 \times 10^{-3}$.

\subsection{Expansion in $\epsilon$}

We seek the solution of Equations $(28)-(38)$ as an asymptotic series in $\epsilon$ :

$$
\begin{array}{ll}
\psi=\psi_{0}+O(\epsilon) ; & \vartheta=\vartheta_{0}+O(\epsilon) ; \\
\chi=\chi_{0}+O(\epsilon) ; & \vartheta^{*}=\vartheta_{0}^{*}+O(\epsilon) ; \\
h=h_{0}+O(\epsilon) ; & \beta=\beta_{0}+O(\epsilon) .
\end{array}
$$

For the leading-order terms, we have the following set of 
equations:

$\Delta\left(\Delta \psi_{0}\right)=0,-\infty<\xi<+\infty, \vartheta_{0}<y<\chi_{0} ;$

$y=\chi_{0}(\xi):\left\{\begin{array}{l}{\left[\frac{\partial^{2} \psi_{0}}{\partial y^{2}}-\frac{\partial^{2} \psi_{0}}{\partial \xi^{2}}\right]\left(1-\chi_{0}^{\prime 2}\right)=4 \chi_{0}^{\prime} \frac{\partial^{2} \psi_{0}}{\partial \xi \partial y} ;} \\ \psi_{0}=1 ;\end{array}\right.$

$y=0, \xi<0: \psi_{0}=0, \frac{\partial \psi_{0}}{\partial y}=0 ;$

$y=\vartheta_{0}(\xi), \xi>0:\left\{\begin{array}{l}{\left[\frac{\partial^{2} \psi_{0}}{\partial y^{2}}-\frac{\partial^{2} \psi_{0}}{\partial \xi^{2}}\right]\left(1-\vartheta_{0}^{\prime 2}\right)=4 \vartheta_{0}^{\prime} \frac{\partial^{2} \psi_{0}}{\partial \xi \partial y}} \\ \psi_{0}=0 ;\end{array}\right.$

$\xi \rightarrow \pm \infty: \psi_{0}<\infty$.

$\left\{\begin{array}{l}\frac{\chi_{0}^{2}}{2}-\frac{\vartheta_{0}^{* 2}}{2 k_{\rho}}=\delta \beta_{0}^{-1} \int_{0}^{\chi_{0}} B_{0} \mathrm{~d} y+\delta \beta_{0}^{-1} \int_{\xi}^{0} \frac{\partial^{2} \psi_{0}}{\partial y^{2}}(\xi, 0) \mathrm{d} \xi, \\ \xi<0: \xi=0: \chi_{0}=1 ;\end{array}\right.$

$\left\{\begin{array}{l}\frac{h_{0}^{2}}{2}+k_{\rho} h_{0} \beta_{0}^{-1} \frac{\mathrm{d} T_{0}}{\mathrm{~d} \xi}-\frac{k_{\rho}}{2} \delta \beta_{0}^{-2}\left(\frac{\mathrm{d} T_{0}}{\mathrm{~d} \xi}\right)^{2}=\beta_{0}^{-1} \int_{\vartheta_{0}}^{\chi_{0}} B_{0} \mathrm{~d} y, \\ \xi>0 ; \xi=0: h_{0}=1 ;\end{array}\right.$

$\vartheta_{0}=\vartheta_{0}^{*}-k_{\rho} h_{0}+\delta \beta_{0}^{-1} k_{\rho} \frac{\mathrm{d} T_{0}}{\mathrm{~d} \xi}, \quad \xi>0 ;$

$\chi_{0}=\vartheta_{0}^{*}+\delta h_{0}+\delta \beta_{0}^{-1} k_{\rho} \frac{\mathrm{d} T_{0}}{\mathrm{~d} \xi}, \quad \xi>0 ;$

$\vartheta_{0}^{*}=k_{\rho}-\delta \beta^{-1} k_{\rho} \frac{\mathrm{d} T_{0}}{\mathrm{~d} \xi}(0), \quad k_{\rho}=1-\delta$,

$B_{0}=B\left(\psi_{0}, \chi_{0}, \xi, y\right), \quad T_{0}=T\left(\psi_{0}, \chi_{0}, \vartheta_{0}, \xi\right)$.

The derived equations describe the ice flow in the transition zone. To order $O(\epsilon)$, the bed slope and the mass accumulation can be neglected. The parameter $\beta_{0}$, as well as the values $L_{0}^{(g)}$ and $A$, is to be determined from matching the near and far fields.

\subsection{Expansions in $\delta$}

Equations (39)-(44) also include the parameter $\delta$, which for the process under consideration is small: $\delta \approx 0.1$. Therefore, the solution to the equations can be sought for as a power series of $\delta$ :

$$
\begin{aligned}
& \psi_{0}=\psi_{00}+O(\delta) ; \vartheta_{0}^{*}=\vartheta_{00}^{*}+\delta \vartheta_{01}^{*}+O\left(\delta^{2}\right) ; \\
& h_{0}=h_{00}+O(\delta) ; \vartheta_{0}=\vartheta_{00}+O(\delta) ; \\
& \beta_{0}=\beta_{00}+O(\delta) ; \chi_{0}=\chi_{00}+\delta \chi_{01}+O\left(\delta^{2}\right) ; \\
& k_{\rho}=1-\delta .
\end{aligned}
$$

Then, for $\psi_{00}$ and $h_{00}$, we have the following set of equations:

$$
\begin{gathered}
\Delta\left(\Delta \psi_{00}\right)=0,-\infty<\xi<+\infty, 1-h_{00}<y<1 ; \\
y=1:\left\{\begin{array}{l}
\frac{\partial^{2} \psi_{00}}{\partial y^{2}}-\frac{\partial^{2} \psi_{00}}{\partial \xi^{2}}=0 ; \\
\psi_{00}=1 ;
\end{array}\right. \\
y=1-h_{00}(\xi), \xi>0: \\
\left\{\begin{array}{l}
{\left[\frac{\partial^{2} \psi_{00}}{\partial y^{2}}-\frac{\partial^{2} \psi_{00}}{\partial \xi^{2}}\right]\left(1-h_{00}^{\prime 2}\right)=-4 h_{00}^{\prime} \frac{\partial^{2} \psi_{00}}{\partial \xi \partial y}} \\
\psi_{00}=0
\end{array}\right.
\end{gathered}
$$

$$
\begin{gathered}
y=0, \xi<0: \psi_{00}=0, \frac{\partial \psi_{00}}{\partial y}=0 ; \\
\xi \rightarrow \pm \infty: \psi_{00}<\infty . \\
\left\{\begin{array}{c}
\frac{h_{00}^{2}}{2}+h_{00} \beta_{00}^{-1} \frac{\mathrm{d} T_{00}}{\mathrm{~d} \xi}=\beta_{00}^{-1} \int_{1-h_{00}}^{1} B_{00} \mathrm{~d} y, \quad \xi>0 ; \\
\xi=0: h_{00}=1 ;
\end{array}\right.
\end{gathered}
$$

Due to Equations $(40)-(44)$, we obtain

$$
\begin{aligned}
\chi_{00}= & 1, \forall \xi ; \chi_{01}=\left[h_{00}-1+\beta_{00}^{-1}\left(\frac{\mathrm{d} T_{00}}{\mathrm{~d} \xi}-\frac{\mathrm{d} T_{00}}{\mathrm{~d} \xi}(0)\right)\right], \\
\chi_{01}= & -\left(\frac{1}{2}+\beta_{00}^{-1} \frac{\mathrm{d} T_{00}}{\mathrm{~d} \xi}(0)\right)+\beta_{00}^{-1} \int_{0}^{1} B\left(\psi_{00}, 1, \xi, y\right) \mathrm{d} y \\
& +\beta_{00}^{-1} \int_{\xi}^{0} \frac{\partial^{2} \psi_{00}}{\partial y^{2}}(\xi, 0) \mathrm{d} \xi, \quad \xi<0 ; \\
& \vartheta_{00}^{*}=1, \vartheta_{01}^{*}=-\left(1+\beta_{00}^{-1} \frac{\mathrm{d} T_{00}}{\mathrm{~d} \xi}(0)\right) ; \\
& \vartheta_{00}=\chi_{00}-h_{00}, \xi>0 . \\
B_{00}= & 4 \frac{\partial^{2} \psi_{00}}{\partial \xi \partial y}+\frac{\partial}{\partial \xi} \int_{y}^{1}\left(\frac{\partial^{2} \psi_{00}}{\partial y^{2}}-\frac{\partial^{2} \psi_{00}}{\partial \xi^{2}}\right) \mathrm{d} y, \forall \xi ; \\
& T_{00}=\int_{1-h_{00}}^{1}\left(\frac{\partial^{2} \psi_{00}}{\partial y^{2}}-\frac{\partial^{2} \psi_{00}}{\partial \xi^{2}}\right) \mathrm{d} y, \forall \xi .
\end{aligned}
$$

Hence, we conclude that $\chi_{00}=1$; in other words, the ice upper surface is, to order $O(\delta)$, a straight horizontal line. It is notable that determination of the functions $\psi, h$ and $\vartheta$ to order $O(\delta)$ permits the determination of $\chi$ and $\vartheta^{*}$ to order $O\left(\delta^{2}\right)$.

Solving Equations (45) and (46) analytically is difficult; therefore, we use computational methods. The algorithm we use requires two initial conditions for $h_{00}$ at the point $\xi=0$. Also, the Equations (45) and (46) include the unknown parameter $\beta_{00}$, whose determination needs another imposed relation. It can be shown (see the Appendix that these conditions are

$$
\xi=0: h_{00}(0)=1, h_{00}^{\prime}(0)=0, h_{00}^{\prime \prime}(0)=0
$$

and there exists a unique solution of Equations (45) and (46).

Ultimately, the set of Equations (45)-(48) defines the unique near-field solution, including a determination of the parameter $\beta_{00}$. Let us call this set the "principal system of equations".

\section{MATCHING PROCEDURE}

5.1. Matching the near-field solution for the transition zone with the far-field one for the glacier grounded part

In order to find the unknown parameter $L_{0}^{(g)}$ for the farfield solution of the grounded glacier part, let us introduce the quantity $\lambda=L_{0}^{(g)} / L_{0}=\lambda_{0}+\delta \lambda_{1}+O\left(\delta^{2}\right)$.

Matching the glacier upper-surface profiles of the farfield solution and the near-field one can be carried out by using the intermediate asymptotic variable $\omega=X / \nu(\epsilon)$, where $\epsilon / \nu \rightarrow 0$ and $\nu \rightarrow 0$ as $\epsilon \rightarrow 0$ (Cole, 1968). In this 
way, all the unknown parameters can be found:

$\lambda_{0}=1$,

$$
\begin{aligned}
\lambda_{1}=- & \left(\frac{1}{2}+\beta_{00}^{-1} \frac{\mathrm{d} T_{00}}{\mathrm{~d} \xi}(0)\right) \\
& +\beta_{00}^{-1} \int_{-\infty}^{0}\left[\frac{\partial^{2} \psi}{\partial y^{2}}(\xi, 0)-\frac{\partial^{2} \psi_{0}^{(g)}}{\partial y^{2}}(0,0)\right] \mathrm{d} \xi .
\end{aligned}
$$

Then, an expression for the ice upper-surface profile $\tilde{\chi}$, which is valid all the way from the divide to the grounding line, can be written in the form

$$
\tilde{\chi}=\frac{L_{0}^{(g)}}{L_{0}}+\chi_{0}-\chi^{(g t)}, \chi^{(g t)}=1+\delta\left(\lambda_{1}-3 \beta_{0}^{-1} \xi\right) .
$$

It should be noted that the parameter $\lambda$ is determined by solving the principal system of equations and is the deviation of the far-field ice thickness at the grounding line from the near-field one there.

\subsection{Matching the near-field solution for the transition zone with} the far-field one for the ice shelf

Matching the far-field ice thickness and the near-field one in the same way as before, we can find the last unknown constant $A=A_{1} \delta^{2 / 3} \epsilon^{4 / 3} \beta_{0}^{1 / 3} / 4$, where $A_{1}$ is determined from the near-field solution. Then, the upper-surface profile and the ice-thickness distribution, which are valid throughout from the grounding line to the ice front, take the forms:

$$
\begin{aligned}
& \tilde{h}=H / L_{0}+h-h^{(s t)}, h^{(s t)}=2 \beta_{0}^{-1 / 2} /\left(\sqrt{A_{1}+\xi}\right) ; \\
& \tilde{\chi}=\frac{L_{0}^{(s)}}{L_{0}}+\chi_{0}-\chi^{(s t)}, \chi^{(s t)}=\vartheta_{0}^{*}+\delta h^{(s t)} .
\end{aligned}
$$

However, since the ice thickness in the ice-shelf region (far from the grounding line) is proportional to $\epsilon$, and the accuracy of the determination of the lower-surface profile is to $O(\delta)$, the elevation of this surface which is valid throughout and is found by the standard procedure as before, will exceed the upper-surface elevation fairly far from the grounding line. For this reason, it is convenient to find the lower-surface profile with formula (42), which is valid everywhere for the floating ice:

$$
\tilde{\vartheta}=k_{\rho}(1-\tilde{h})+\delta \beta_{0}^{-1}\left(\frac{\mathrm{d} T_{0}}{\mathrm{~d} \xi}-\frac{\mathrm{d} T_{0}}{\mathrm{~d} \xi}(0)\right) .
$$

\section{OVERALL ALGORITHM FOR SOLVING THE PROBLEM}

First, it is necessary to solve the principal system of equations. As a result, the functions $\psi_{00}, h_{00}$ and the parameter $\beta_{00}$ can be found. The functions $\chi_{0}, \vartheta_{0}^{*}$ and $\vartheta_{0}$ are determined to various orders of accuracy by the formulae in Equations (47).

Let us move the origin of the coordinate system to the bed at the divide, whose position is known. Then the point $x=x_{\mathrm{d}}$ defines the grounding line. Knowing $\beta$, we can find the length $x_{\mathrm{d}}$ of the grounded glacier part from the equation

$$
\beta-\frac{\delta\left(z^{*}-z_{0}\left(x_{\mathrm{d}}\right)\right)^{3} \rho_{\mathrm{i}} g}{q\left(x_{\mathrm{d}}\right) \eta \vartheta^{* 3}}=0
$$

which is a consequence of Equation (35). In Equation $(50)$, the function $z_{0}(x)$ is known, because the bed profile is given and $q(x)=\int_{0}^{x} f^{+} \mathrm{d} x$.

Having determined $x_{\mathrm{d}}$, the water depth $z^{*}$ becomes known as well as the scales of non-dimensionalization. Then, we can find the scaled ice thickness at the grounding line:

$$
L_{0}=\frac{z^{*}-z_{0}\left(x_{\mathrm{d}}\right)}{[z] \vartheta^{*}} .
$$

Knowledge of the value of $\lambda_{1}$, which is found from Equation (49), allows us to determine the glacier uppersurface profile far from the grounding line by solving Equation (26) with the initial condition: $X=0: L_{0}^{(g)}$ $=\lambda L_{0}$.

The problem for the ice shelf has an analytical solution. The constant $A$ is known from the near-field solution. Then, it is easy to construct glacier-surfaceprofile distributions, which are valid throughout the region. Although the ice-upper-surface elevation is found to be $O(\delta)$, its gradient can be determined as $O\left(\delta^{2}\right)$.

It should be noted that Salamatin's (1984) boundary condition can be transformed to the form found in this paper by choosing the proper value of the parameter

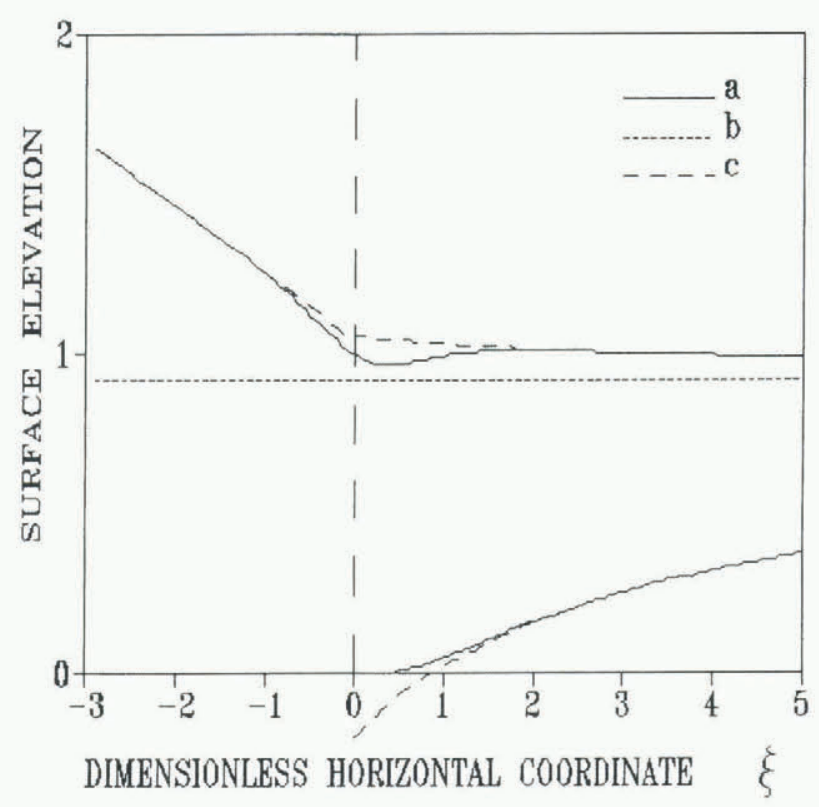

Fig. 2. The near-field solutions (a) for the glacier surface profiles differ from the far-field ones (c) for $-1<\xi<2$. There is a point of local minimum of the upper-surface elevation. The dashed line $(b)$ is the water level. 
which characterizes the deviation of the reduced normal deviatoric stress from its true value. Comparison of the different boundary conditions, as well as numerical methods, to solve the near-field problem will be considered elsewhere. The results of calculations are as follows:

$$
\begin{aligned}
& \beta_{0}=1.5+O(\delta), \quad \vartheta_{0}^{*}=1-0.67 \delta+O\left(\delta^{2}\right), \\
& \lambda=1+0.32 \delta+O\left(\delta^{2}\right), \quad A_{1}=1.86 .
\end{aligned}
$$

The glacier-surface profiles are shown in Figure 2, in which we can see that the upper-surface elevation has a point of local minimum, which coincides with the conclusion of Barcilon and MacAyeal (1993). For comparison, the far-field solutions, as $\xi \rightarrow 0$, are shown. It is clear that, at a distance of several ice thicknesses from the grounding line, the far-field solutions properly describe the ice dynamics.

Several tests on the model examples were carried out to compare the grounding-line positions found using Thomas and Bentley's (1978) boundary conditions and those derived in this paper. The difference between the results obtained is about $10 \%$ of the grounded glacier length.

\section{CONGLUSIONS}

The study carried out here allows us to find strict boundary conditions at the grounding line without the use of simplifying assumptions, such as are made in the boundary-layer approximation. The parameter $\beta$ is determined by the solution of the near-field problem; however, for real glaciers, it can be found by natural surveys. Changes in this parameter should reflect individual peculiarities of the specific glacier, such as sliding, temperature distributions and bed roughness.

The important result lies in the fact that the near-field ice thickness at the grounding line and that of the far field for the grounded glacier part differ from the flotation thickness by $-3.3 \%$ and $-0.1 \%$, respectively. This means that the condition of hydrostatic equilibrium for the ice at the grounding line is the boundary condition for the grounded part of the glacier as well as being a proper approximation for the relationship between the ice thickness and the water depth at that line.

The glacier-surface profiles in the vicinity of the grounding line can be found separately from the solutions of the far-field problems, where the span scales are much higher. This allows us to find the glacier-surface elevation to a sufficient order of accuracy in all regions. The existence of a local minimum of the upper-surface elevation almost over the grounding line is commensurate with real glacier characteristics. This implies that the detection of such a line of local minimum elevation by surveys can point to the grounding-line position.

Our coherent approach to the study of all parts of the glaciological system (grounded ice sheet, ice shelf and transition zone) allows us to find relationships between the spatial scales of its parts via the parameters $\epsilon$ (typical ice-surface slope) and $\delta$ (normalized difference between ice and water densities), which characterize exterior conditions of the existence of the glacier. This also allows us to construct a complete model describing the dynamics of the system.

Despite the fact that most glaciers are essentially nonisothermal, the form of the boundary conditions at the grounding line does not change, except for the constants $\beta$ and $\vartheta^{*}$. The case of Glen's flow law will be considered elsewhere.

\section{ACKNOWLEDGEMENTS}

We are grateful to Professor Salamatin for his remarks and for drawing our attention to the problem. We are indebted to Dr A. Fowler for useful discussion that revealed several inaccuracies. We also appreciate the help of Professor Karchevsky in regard to calculation methods. This work would have been impossible without the financial assistance of the European Science Foundation and the Russian Fund of Fundamental Research (grant No. 94-01-01191a).

\section{REFERENGES}

Barcilon, V. and D. R. MacAyeal. 1993. Steady flow of a viscous ice stream across a no-slip/free- slip transition at the bed. F. Glaciol., 39 (131), 167-185.

Cole, J. D. 1968. Perlurbation methods in applied mathematics. Waltham, MA, etc., Blaidsell Publishing Co. Division of Ginn and Co.

Fowler, A. C. 1981. A theoretical treatment of the sliding of glaciers in the absence of cavitation. Philos. Trans. R. Soc. London, Ser.A, $298(1445), 637-685$.

Hutter, K. 1983. Theoretical glaciology; material science of ice and the mechanics of glaciers and ice sheets. Dordrecht, etc., D. Reidel Publishing Co. Tokyo, Terra Publishing Co.

Hutter, K. 1993. Thermo-mechanically coupled ice-sheet responsecold, polythermal, temperate. J. Glaciol., 39(131), 65-86.

Lestringant, R. 1994. A two-dimensional finite-element study of flow in the transition zone between an ice sheet and an ice shelf. Ann. Glaciol., 20, $67-72$.

Lingle, C. S. 1984. A numerical model of interactions between a polar ice stream and the ocean: application to Ice Stream E, West Antarctica. J. Geophys, Res., 89 (C3), 3523-3549.

Salamatin, A. N. 1984. O dvigenii kraya pokrovnogo lednika [Motion of the edge of an ice sheet]. Issled. Prikladnoy Matematike, 10, 150-155. [Translation in J. Sov. Math., 44(5), 672-675 (1989).]

Salamatin, A. N. and A. B. Mazo. 1984. Issledovaniye obschey matematicheskoy modeli kupilovidnogo lednika metodami teorii podobiya [Similarity analysis of the general mathematical model of an ice-cap glacier]. Issled. Prikladnoy Matematike, 10, 139-149. [Translation in J. Sov. Math., 44(5), 664-672 (1989).]

Sanderson, T.J. O. 1979. Equilibrium profile of ice shelves. $\mathcal{J}$. Glaciol., $22(88), 435-460$.

Thomas, R. H. and C. R. Bentley. 1978. A model for Holocene retreat of the West Antarctic ice sheet. Qual. Res., 10(2), 150-170.

Van der Veen, C.J. 1983. A note on the equilibrium profile of a free floating ice shelf. Utrecht, Rijksuniversiteit Instituut voor Meteorologie en Oceanografie. (Technical Report 83(15).)

Weertman, J. 1957. Deformation of floating ice shelves. J. Glaciol., 3(21), $38-42$.

\section{APPENDIX}

The numerical algorithm to solve Equations (45) and (46) requires two initial conditions for $h_{00}$ at the point $\xi=0$. In addition, Equations (45) and (46) include the unknown parameter $\beta_{00}$, whose determination needs another imposed condition. In order to elucidate these 
questions, it is necessary to examine the behaviour of the function $h_{00}$ as $\xi \rightarrow 0$ and $\xi \rightarrow+\infty$.

The asymptotic behavior of $\psi_{00}$ and $h_{00}$, when $\xi \rightarrow$ $+\infty$, can easily be derived:

$$
h_{00}=\frac{2 \beta_{00}^{-1 / 2}}{\sqrt{A_{1}+\xi}}, \quad \psi_{00}=\frac{(y-1)}{h_{00}}, \quad \xi \rightarrow+\infty .
$$

Generally, $A_{1} \neq A$. From Equations (51), we can conclude that

$$
\lim _{\xi \rightarrow+\infty} h_{00}=0
$$

We now convert Equation (46) to a form from which we can deduce the behaviour of $h_{00}$ in the vicinity of the grounding line. With the use of the first equation of (12) and after differentiation, Equation (46) can be transformed to the following form:

$$
\left\{\begin{array}{c}
\beta_{00} h_{00}^{\prime}+h_{00}^{\prime \prime}\left[\frac{\partial^{2} \psi_{00}}{\partial y^{2}}-\frac{\partial^{2} \psi_{00}}{\partial \xi^{2}}\right]_{y=1-h_{00}}-h_{00}^{\prime 2}\left[\frac{\partial^{3} \psi_{00}}{\partial y^{3}}-\frac{\partial^{3} \psi_{00}}{\theta \xi^{2} \partial y}\right]_{y=1-h_{00}} \\
+2 h_{00}^{\prime}\left[\frac{\partial^{3} \psi_{00}}{\partial y^{2} \partial \xi}-\frac{\partial^{3} \psi_{00}}{\partial \xi^{3}}\right]_{y=1-h_{00}} ; \\
=\left[3 \frac{\partial^{3} \psi_{00}}{\partial \xi^{2} \partial y}+\frac{\partial^{3} \psi_{00}}{\partial y^{3}}\right]_{y=1-h_{00}} ; \\
\xi=0: h_{00}=1
\end{array}\right.
$$

For the problem under consideration, we assume that $h_{00}$ is a smooth function and belongs to the class $H^{2}(-\infty,+\infty)$; then we have $h_{00}^{\prime}(0)=0$. Taking, in the vicinity of the grounding line $h_{00}(\xi) \approx 1$, we can find $\psi_{00}$ when $\xi$ and $y$ are small (Barcilon and MacAyeal, 1993): $\psi_{00}=C r^{3 / 2} \cos (\theta / 2) \sin \theta+O\left(r^{5 / 2}\right)$, where $r$ and $\theta$ are polar coordinates and $C$ is an unknown constant. It is easy to verify that, with the stream function chosen in this way, $h_{00}(\xi) \equiv 1$ will be the solution of Equation (53). Also, the coefficient of $h_{00}^{\prime \prime}$ tends to zero, as $\xi \rightarrow 0$. These results suggest that complementary initial conditions are defined by the problem itself and are $h_{00}^{\prime}=0, h_{00}^{\prime \prime}<\infty$ on $\xi=0$.

Generally, the solution of Equation (53) with arbitrary $\beta_{00}$ does not satisfy condition (52), which is necessary to match the far-field solution for the ice thickness to the near-field one, because it was derived from Equation (46) by differentiation. Hence, we have condition (52) to determine $\beta_{00}$. However, if we solve Equations (45) and (46), then Equation (52) is satisfied, but the relation $h_{00}^{\prime \prime}(0)<\infty$ is not valid for arbitrary $\beta_{00}$. Numerical calculations show that this relation is equivalent to $h_{00}^{\prime \prime}(0)=0$ and numerical approximation of $h_{00}^{\prime \prime}(0)$ with finite $\Delta \xi$, where $\Delta \xi$ is the discretization length, is a continuous function of $\beta_{00}$. Hence there exists a unique solution of Equations (45) and (46). 